\title{
A SIMPLE METHOD FOR A FOURIER ANALYSIS
}

\author{
by J. M. W. MILATZ, A. H. WAPSTRA *) and \\ J. S. VAN WIERIN(GEN †)
}

Physisch Laboratorium der Rijksuniversiteit Utrecht, Nederland

\section{Synopsis}

A Fourier analysis has been made using only a phototension cell, an alternating current galvanometer and a rotating device.

1. Introduction. During the course of experiments ${ }^{\mathbf{1}}$ ), in which we made recordings of the fluctuations of an electrometer influenced by mechanical perturbations, it became necessary to obtain a Fourier analysis of some of these recordings. For this analysis an arrangement was made, founded on the great selectivity of the alternating current galvanometer, being a galvanometer in which the magnetic field is substituted by a $50 \mathrm{~s}^{-1}$ alternating magnetic field. As a result of this alternation the galvanometer is only sensitive to alternating currents having a frequency very close to $50 \mathrm{~s}^{-1}$.

If the curve is converted into a fluctuating current through the galvanometer, this galvanometer will only respond to the components of this current near the frequency of its field.

This idea could be realised in a simple way. The curve to be analysed was copied on black paper, cut out and bent into a cylinder. This cylinder was rotated with an adjustable speed between a slit $S$ illuminated by a 25 watt incandescent lamp and a barrier layer photoelectric cell $P$ (fig. 1). This cell gave enough voltage for the galvanometer $G$.

As the equation of the galvanometer is linear, its deviation under the influence of the fluctuating current can be described by expanding this current in a Fourier series; the total displacement of

*) Now at the „Instituut voor Kernphysisch Onderzoek”, Amsterclam

$\dagger$ Present address: Philips Research Laboratories, Eindhoven. 
the coil is the sum of the displacements due to the separate Fourier components. If the amplitude of the $n$-th component of our curve is $\varphi_{n}$, the $n$-th component of the current is proportional to $\varphi_{n}$. If the curve completes one revolution in $T$ seconds, the frequency of the $n$-th component is $n T^{-1} \mathrm{~s}^{-1}$, so:

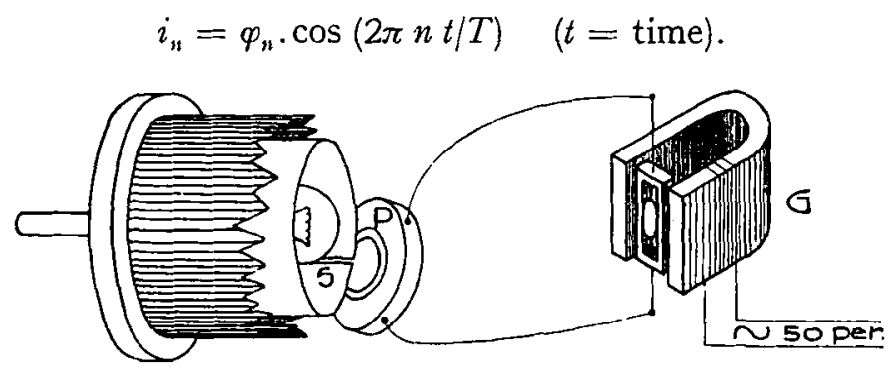

Fig. 1. Experimental arrangement.

The field of the galvanometer is

$$
H=H_{0} \cos 2 \pi \nu_{1} t
$$

and the torque $M$ on the coil is proportional to the product of these quantities, so that:

$$
M=\varphi_{n} H_{0} \cos 2 \pi\left(v_{1}-n T^{-1}\right) t+\varphi_{n} H_{0} \cos 2 \pi\left(v_{1}+n T^{-1}\right) t
$$

The first term is a torque with constant amplitude but varying frequency. If the coil of the galvanometer has a natural frequency $v_{0}$ and is aperiodically damped, then this torque will give a forced vibration with a frequency:

$$
v=\nu_{1}-n / T
$$

and an amplitude proportional to:

$$
a \sim \varphi_{n} H_{0}\left(1+\left(v / v_{0}\right)^{2}\right)^{-1}
$$

For the second term in (1) we get the same result, only the frequency becomes $\left(v_{1}+n / T\right)$. Now each torque gives only a measurable result if the frequency is low compared to $v_{0}$. Because $\nu_{1}$ is much larger than $v_{0}$ we can neglect the second term.

In order to analyze the movement of the galvanometer we consider the case that only one Fourier component is present. At the start the curve is rotated at a rather low speed: $n T^{-1}<v_{1}$. If we 
increase the rotation speed, the frequency (2) decreases and the amplitude is maximum; by further increasing the rotation speed the frequency increases and the amplitude decreases. The variation of the amplitude with the rotation speed is shown in fig. 2 for our galvanometer, where the frequency of the field was $\nu_{1}=50 \mathrm{~s}^{-1}$ and the natural frequency of the coil $v_{0}=0.32 \mathrm{~s}^{-1}$.

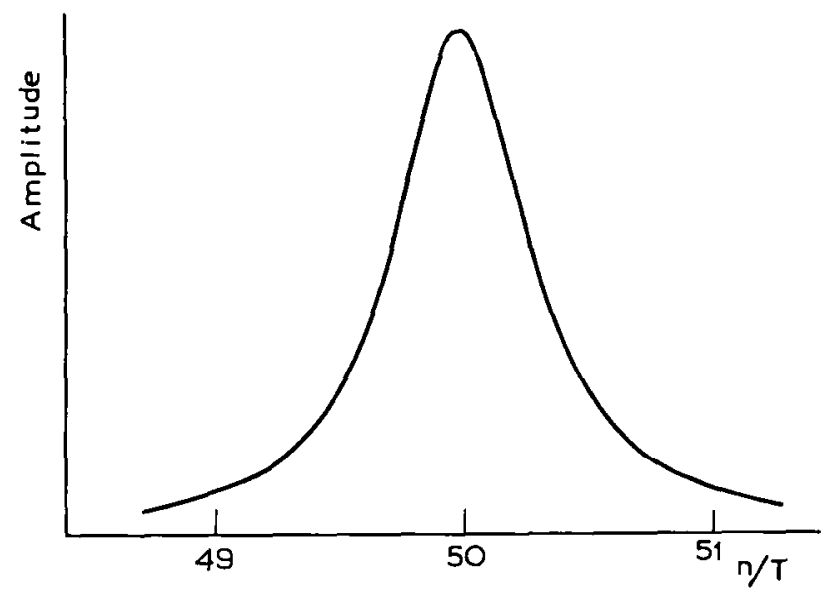

Fig. 2. Dependence of the oscillation amplitude of the galvanometer on the rotation speed of the curve to be analyzed.

We could follow this course very well with a pure sine wave. If more Fourier components are present, we must add the deviations due to each of them.

2. Measuring method. The Fourier components actually to be measured were in the neighbourhood of $n=30$. Because in the beginning of our experiments the frequency of our coil was $v_{0} \simeq 1$ $\mathrm{s}^{-1}$, the amplitude of the nearest components, if the 30-th component was in resonance, was about $\frac{1}{3}$ of its amplitude in resonance. Hence the galvanometer was not selective enough to measure the components separately. It was therefore tried to increase the moment of inertia of the coil. Because we wanted to measure with an aperiodically damped galvanometer the damping should be increased too. The moment of inertia was therefore increased by a circular copper disc, which could be placed in a magnetic field, for increasing the damping too (fig. 3). By means of this method the frequency of the movement of the undamped coil was decreased to $v_{0}=0.3 \mathrm{~s}^{-1}$. 
Even with the 50-th Fourier component resonating, the amplitude due to the 51 -th is now only $8 \%$ of its amplitude in resonance. We checked this with a curve constructed by adding a 50-th and 51-th component only.

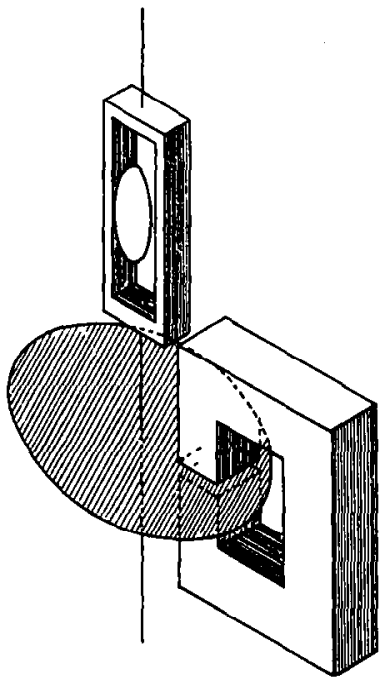

Fig. 3. Galvanometer provided with increased moment of inertia.

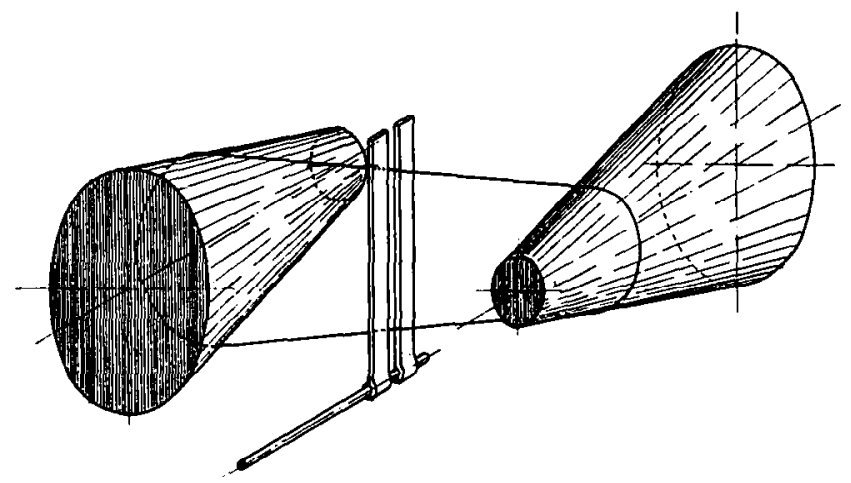

Fig. 4. Arrangement to control the turning speed.

In order to get a fairly constant turning speed of the curve, that could easily be controlled, we used a synchroneous electromotor and a gear box. The latter we improvised by using two conical wheels and a belt, which could be moved in the direction of the axis of the wheels (fig. 4). With this construction the turning speed could be 
controlled very easily. The constancy was not very high, because the position of the belt on the wheels was not stationary but oscillated slightly. It is not difficult to make a better arrangement, but for our purpose it was good enough.

The width of the slit $S$ (fig. 1) must be adjusted in such a way that the highest component to be measured is not disturbed. In our case this was about the 40-th component. As the circumference of our curve was about $16 \mathrm{~cm}$, the corresponding wavelength was about $4 \mathrm{~mm}$. Therefore a slit of width $1 \mathrm{~mm}$ was used.

With this arrangement the curve shown in fig. 5 was analyzed, being the recording of the fluctuation of our electrometer (Brownian motion and microseismic perturbation $\left.{ }^{1}\right)$ ).

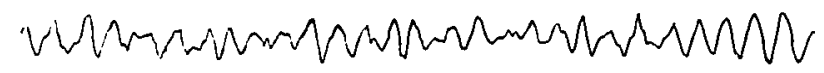

Fig. 5. Analysed curve (from a recording of a fluctuating electrometer).

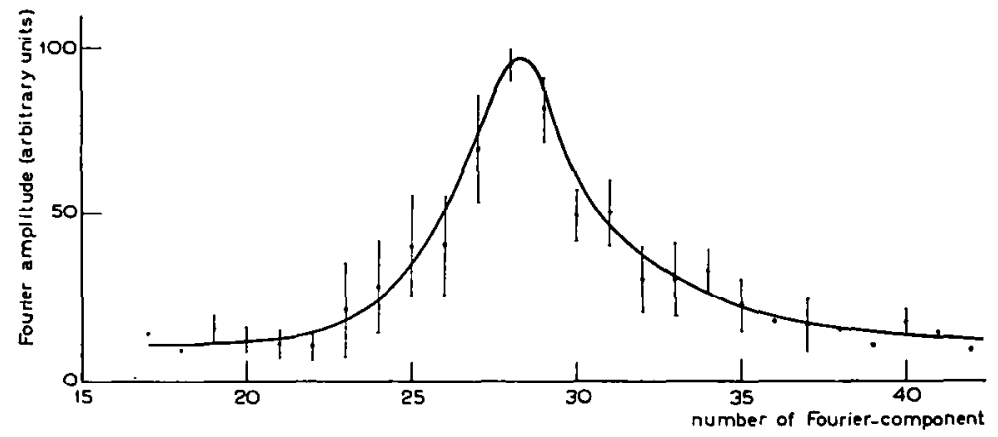

Fig. 6. Fourier analysis of the curve shown in fig. 5 . The vertical lines give the maximum deviations of the amplitudes found for components which have been measured twice or more.

Fig. 6 shows the result of 4 independent measurements. The given Fourier amplitudes are the largest amplitudes of the motion of our galvanometer in $\mathrm{mm}$. The rather large fluctuations in the amplitudes must be described to the variation of the turning speed. Our analysis gives a discrete Fourier spectrum, because it is a repeated analysis of a part of the recording. The continuous Fourier spectrum of an infinite recording is expected to be represented fairly well by a curve, drawn smoothly through the points of fig. 6 . 
3. Possible improvements. No efforts were made to improve the arrangement, because our purpose was to get only an idea of the mean course of the Fourier spectrum of our curve.

If we want to improve it, first of all the constancy of the turning speeds should be increased. But it is also possible to make an automatic arrangement. This can be done by replacing the motor by a driving wheel ${ }^{2}$ ). After having set this wheel turning at a predetermined speed it is left to itself. Then its turning speed will decrease gradual$1 y$, and one after another the frequencies of the Fourier spectrum will pass the frequency of the galvanometer. As explained in section 1 the motion of the coil of the galvanometcr will assume the form of fig. 7. We can make a recording of this motion. On this recording the maximum amplitudes give us the Fourier amplitudes, and by counting we can easily get the ordinal numbers of the measured components.

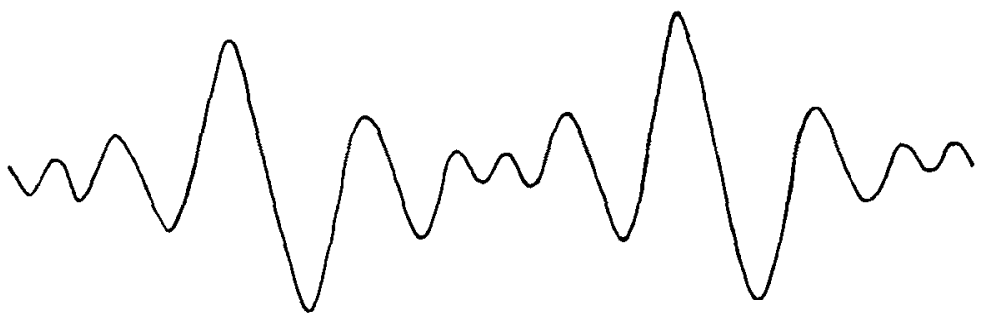

Fijg. 7. Hypothetical form of an autonatic recording of the Fourier components.

With our galvanometer the time of measurement would have to be rather long: about one hour. It may be shortened by replacing our galvanometer, designed for a $50 \mathrm{~s}^{-1}$ alternating current, by onc designed for a higher frequency. With $500 \mathrm{~s}^{-1}$ the time of measurement could be shortened ten times. Then the whole spectrum of our curve would be obtained within about 10 minutes.

Received 16-10-52.

REFIERENCES

1) M ilat $z$, J. M. W. and Wapstra, A. H., Physica 1! (1953, $1 \leq 6$.

2) Barber, N. F., Ursell, F., I) arbyshirc, J. and Tucker, M. J., Nature 1.58 (1946) 329. 